\title{
Issues and Challenges of using Blockchain for IoT Data Management in Smart Healthcare
}

\author{
Gerasimos Vonitsanos ${ }^{1}$, Theodor Panagiotakopoulos ${ }^{2}$ and Andreas Kanavos ${ }^{2,3 *}$ \\ ${ }^{1}$ Computer Engineering and Informatics Department University of Patras, Patras, Greece \\ ${ }^{2}$ School of Technology and Science Hellenic Open University, Patras, Greece \\ ${ }^{3}$ Department of Digital Media and Communication Ionian University, Kefalonia, Greece
}

*Corresponding author: Andreas Kanavos, School of Technology and Science Hellenic Open University, Patras, Department of

Digital Media and Communication Ionian University, Kefalonia, Greece

\section{ARTICLE INFO}

Received: 㓞 November 19, 2021

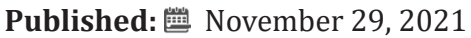

Citation: Gerasimos Vonitsanos, Theodor Panagiotakopoulos, Andreas Kanavos. Issues and Challenges of using Blockchain for IoT Data Management in Smart Healthcare. Biomed J Sci \& Tech Res 40(2)-2021. BJSTR. MS.ID.006423.

\begin{abstract}
The features provided by Blockchain technologies such as decentralized storage, advanced security, and authentication indicate that they can be used in other applications such as the healthcare sector. The 1996 Health Insurance Portability and Accountability Act (HIPAA) legal requirements stipulate strict authentication when sharing files in the healthcare sector. It is therefore essential to further develop blockchain technology, and through this work, the benefits of using these technologies in healthcare are highlighted.
\end{abstract}

Keywords: Blockchain; e-Health; Internet of Things; Healthcare Data Management; IoT Data Management; Security; Smart Cities; Healthcare

\section{Introduction}

The agile advancement of technologies utilizing the Internet of Things (IoT) has significantly improved health- related services such as electronic drug prescriptions as well as Electronic Health Records (EHR) insurance information [1,2]. The collection of patients' medical data can be made more accessible using IoT technologies by improving workflow automation. As a result, meaningful information about patients' symptoms is provided immediately, facilitating their treatment and remote monitoring in real-time [3,4]. A significant advantage is that it is possible to monitor patients from home via wireless sensors, thus reducing the need for regular hospital visits [5]. Furthermore, these medical devices can send alerts if something serious happens [6-8]. Also, the patient's surrounding area can be monitored, and, through data processing, health conclusions can be drawn [9]. The volume of data stored in EHRs has increased significantly through the use of IoT devices located in different locations, and managing this data is extremely difficult [10]. Cyberattacks have also increased as most medical systems are vulnerable to a single point of failure resulting in information leakage with severe consequences [11].

Many EHR management systems do not provide transparency and privacy control, nor are there advanced security mechanisms [12]. Blockchain technology could provide solutions to many problems in medical health systems [13,14]. As mentioned in [15] blockchain technologies can also be integrated into smart cities and smart homes. Several organizations have been interested in Blockchain technology and specifically in bitcoin digital cryptocurrency. Transactions with transparency and verification mechanisms can be securely stored through the blockchain. Stakeholders in blockchain technology can trade securely without Intermediators [16-21]. In areas such as the automotive industry, aerospace, banks, and defense agencies, significant changes have occurred from smart technologies and the blockchain [22,23]. As the volume of passengers and flights is constantly increasing, a technological solution to improve the management processes 
will include IoT systems that will be interconnected with other platforms and will implement the concept of smart airports [24] As the size and complexity of patient health record files continue to grow, blockchain technologies have aided all stakeholders in communicating. However, there is still a long way to go due to the variations of different networks [25].

In addition, if unauthorized users have access to patient data, then there is a risk of criminal activities, highlighting the need to ensure the confidentiality of patient data [26]. Blockchain technologies help in this direction, providing the integrity of the data and preventing their violation [27]. Several researchers have studied the effect of using blockchain technologies [28-31,14,32] in the healthcare sector. Their research focuses on how privacy and security in file-sharing should be improved between stake- holders through blockchain technologies. However, preventingunauthorized users and interoperability in file-sharing in healthcare applications require specific criteria that may not be fully addressed due to demanding legal requirements. Using blockchain technologies, smart contracts, and access control technologies, state-of-the-art healthcare delivery in a smart city can be improved. Each patient's EHR can be stored securely without any privacy issues. Generally, in these cases, there is a need for access from different departments to information in the stored medical history of patients [33]. The prevention of counterfeiting can be achieved by managing the supply chain of medical products through blockchain as medical products are detected and tested for their origin [34].

\section{Recent Advances of Blockchain Applications in Healthcare}

Blockchain technologies contain, apart from others, Cryptography, economic models, Mathematics, and Algorithm. To solve widespread distributed database synchronization problems, a combination of distributed consensus algorithms is used; hence it is not just a single technique but an integrated infrastructure construction in multiple fields [35-37].

Blockchain technologies consist of six key elements.

1. Decentralization: The data is stored and updated distributively since the blockchain is not originating from a centralized node.

2. Transparency: The records of data are transparent from one node to another in a blockchain system

3. Open Source. The source code of a blockchain system is public, and anyone uses blockchain technologies.

4. Autonomy: The main concern of a blockchain system is to ensure that the data is transferred safely. Therefore there is no intervention between the single users and the whole system between the nodes.
5. Immutable: The data records cant be transformed unless a user controls over $51 \%$ of the node simultaneously.

6. Anonymity: The transactions between trusted nodes can be anonymous using only blockchain addresses.

Several experiments and relevant literature highlight the many possibilities that Blockchain has for healthcare technology. Some software solutions will be discussed in the following subsections.

\section{Healthcare Data-Sharing through the Gem Health Network}

When a patient needs to be treated in a different hospital, confidential records should be shared, especially when specialized treatment is in another country. Healthcare-related files include many documents that are confidential and fall under strict laws that are different in each country, such as the 1996 Health Insurance Portability and Accountability Act (HIPAA) [38,39]. At each patient visit to a different doctor, new medical records need to be created. The corresponding data will be exchanged between professionals, and the medical records need to be updated with the latest data. Authentication between different electronic platforms may take a great deal of time to process the information for all stakeholders [34]. Gem Health Network using Blockchain Ethereum technology could solve the problem as mentioned earlier [34]. This network gives healthcare professionals access to the necessary medical data without any centralized restrictions on storage. Therefore, users who have the authorization required can search for data in medical records in real-time, reducing the risk of making an error in diagnosis due to outdated information.

\section{OmniPHR}

The OmniPHR framework has been developed to make it easier to manage patients' health records, as it provides the necessary grouping, regardless of whether they are located in many different healthcare providers [40]. As a result, healthcare providers now have access to up-to-date patient data, without being hindered by the distribution of data in many different systems. The difference between EHR and Personal Health Records (PHR) is a significant problem OmniPHR should address. Initially, through specific government standards, an effort is made to keep EHR records uniform in all countries and to keep them up to date. Doctors inform them without interacting with patients, which is the main difference with PHR files, as the later are created managed by patients. OmniPHR provides a framework for completing patient records with the level of accuracy required by the medical community. OmniPHR incorporates blockchain technology specifically for the interoperability of PHR files to provide a unified view of health records. Specifically, OmniPHR seeks to improve a distributed architecture in terms of scalability and interoperability. 


\section{Medrec}

Medrec uses blockchain technology to manage Electronic medical records (EMRs). which comprises instances of EHRs, on a decentralized file management system [41]. As a result, patients' medical information is accessible simultaneously to different medical providers, with features such as authentication and data sharing following the principles of confidentiality and accountability. MedRec focuses more on the analysis of the approach and implementation of the framework before any field tests. This solution favors interoperability between patients and doctors. As blockchain transactions need to have a group of miners, the MedRec system suggests that medical stakeholders participate as miners. They will have access to anonymous data as a reward for maintaining network security. In this way, patient data can take the form of metadata. The smart contracts that Medrec consists of help automate and keep track of changes such as adding new records or changing access rights. The Ethereum blockchain implementations provide the patient-provider relationship, with smart contracts linking medical history with permissions and data retrieval methods. All information is encrypted, and authorizations from patients are made only through providers.

\section{Virtual Resources}

The Virtual resources are capable of shifting the load distribution on edge hosts and facilitating multi-tenancy support. They can be used as a software-defined IoT management construct [42]. Specific problems that can be solved on IoT devices that can be solved are

1. Lack of a mechanism that will facilitate the secure distribution of the software on the servers

2. Lack of a mechanism for controlling the access of the software

3. No adequate support for virtualization issues.

\section{Benefits of Blockchain Technology in Healthcare Data Management}

This section outlines the main benefits of leveraging blockchain technology for healthcare data management systems [43].

\section{Accuracy of Medical Data}

To obtain a patient's complete medical history, all the data must be collected in an automated way, as they are fragmented in different medical centers and insurance companies. Through the storage of all patients' medical data in a blockchain, there will always be an update in information and automatic detection for any violations [44]. As a result, healthcare professionals can provide more effective treatments through blockchain technologies, as a complete view of the medical history is available and all data is secure and immutable [28].

\section{Medical Data Interoperability}

Most medical systems with EHR data are from different manufacturers with different technical specifications $[45,46]$. Therefore, sharing data in the same format is challenging to achieve as the platforms have many technical differences. To obtain interoperability between two EHR systems, standardization in the data encoding should be applied [47]. Thus, there is a limit to the electronic information exchange as the data do not have the necessary standardization. However, through blockchain technologies, this limitation can be overcome, as all EHR data stored will follow a specific standardization.

\section{Security of Medical Data}

Several cyberattacks on healthcare organizations could have been undertaken [48]. The systems for handling digital medical records used by several healthcare industries operate manually, so they are already quite outdated. Therefore, users can easily modify medical records without the necessary authorization. As a result, medical data can be lost permanently by human mistakes. Using Blockchain technologies can help prevent medical data from being altered by unauthorized users or by human mishandling. In addition, in case of natural disasters, the data is safe as it is stored in different locations of Blockchain.

\section{Costs of Handling Medical Data}

The handling cost is relatively high in healthcare systems in everything related to patient data, as patients' medical records are located in different health facilities. Therefore, collecting all the data manually leads to high costs due to the long delay in the process [49]. Using Blockchain technologies can help reduce this cost for medical companies as they can access complete patient data without having to collect it from different locations [50].

\section{Global Access of Medical Data}

In some cases, healthcare professionals should be fully aware of the medical history of patients [51] before prescribing any medication. This way, they will analyze various aspects of the medical history, information about any allergies, and choose the appropriate treatment. Unfortunately, most health- care management systems do not have this option as global access to medical data [52] is not allowed.

\section{Data Audits in Healthcare Industries}

The audit process in the healthcare industry is necessary to assess whether specific procedures are followed in their daily operations. However, healthcare data management systems are vulnerable to breaches and operate manually, blocking the audit processes. Blockchain technologies will help provide reliability to audit procedures as it secures data in ways of authentication and 
immutability. As a result, healthcare services will be upgraded, and there will be compliance with the necessary legal regulations.

\section{Challenges of Using Blockchain Technologies in Healthcare-Derived IOT}

This section introduces several fundamental open challenges that can prevent the utilization of the corresponding blockchain technology regarding the healthcare sector.

\section{Interchange of Information}

In blockchain networks regarding the healthcare sector, exchanging information between different stakeholders plays a vital role. Those interested could be the departments of the same hospital or other, insurance companies, and specialized staff such as doctors. However, ensuring the proper exchange of information is a challenge due to the diversity of the parties involved [30].

\section{Privacy Leakage and Security}

Although the decentralization method is secure, there are several disadvantages if a privacy leakage of data distribution in the public ledger occurs. In a blockchain environment, there is trust between stakeholders regarding data sharing as they trust each other. But in some cases, such as if $51 \%$ of nodes is infected, this scenario fails [30].

\section{Difficulties of Storage Requirements}

There are difficulties in maintaining patients' medical records as they consist of many documents and images. There- fore, their size is enormous, and they need substantial storage spaces. In addition, sharing data due to medical transactions means that the data may be distributed across multiple locations or stored more than once in the same format. Therefore, the healthcare system will undoubtedly be affected by the specific difficulties of providing colossal storage spaces [53].

\section{Standardization of Protocols and Technologies}

Blockchain is already widely used worldwide in applications where security and trust are required. It is therefore essential that the technologies and protocols to be used are correctly standardized. In addition, data-related issues, in particular their size and format, should also be defined in detail from the start $[51,54]$.

\section{Building Trust for Sharing the Medical Records Among Hospitals}

Hospitals usually do not want to share their patients' medical records as they will have to change their pricing policy because they charge their patients differently. The same goes for insurance companies that do not want to share medical data, as they are related to fees. Therefore, stakeholders should be persuaded to share their data once trust has been built between them for a better healthcare system [55].

\section{Building Trust for Sharing the Medical Records}

In the public domain, many patients do not want to share their medical records with third parties; therefore, building trust between them must be achieved. More specifically, the effort to build trust and confidence in privacy and security among the patients should be strengthened in a healthcare system based on Blockchain and the IoT.

\section{The Challenge of E-Prescribing}

Differentiating electronic prescriptions from paper prescriptions is undoubtedly a massive challenge for doctors. Usually, doctors fill in only the necessary information on paper forms, but they will not ignore them in the electronic files. So an essential element related to the success of healthcare based on Blockchain technology is the proper training of doctors. Therefore, they must have developed the required skills and been adequately trained to trust the new technologies and use them.

\section{Data Ownership - Rules and Processes}

A big challenge is the data ownership plan that establishes accountability and responsibility in the stages of data creation to data consumption. For example, a proper data ownership plan should be created in the healthcare sector to consider the following core elements: Management, Location, Access, Security and Rights and Retention.

\section{Conclusion and Future Work}

This research addresses the various gaps in blockchain technologies in the healthcare industry. Much research has already been done on the use of blockchain in the healthcare industry, and we have included the results of this research. Using blockchain technologies in the healthcare industry can solve many difficulties in the security of files that need to be shared, but this does not mean that we can apply it in any case. Therefore, there should always be an assessment before using these technologies about how they affect health services.

\section{References}

1. Griggs KN, Ossipova O, Kohlios CP, Baccarini AN, Howson EA, et al. (2018) Healthcare blockchain system using smart contracts for secure automated remote patient monitoring. Journal of Medical Systems 42(7): 130.

2. Ray PP, Dash D, Salah K, Kumar N (2021) Blockchain for iot-based healthcare: Background, consensus, platforms, and use cases. IEEE Systems Journal 15(1): 85-94.

3. Tao H, Bhuiyan MZA, Abdalla AN, Hassan MM, Zain JM, et al. (2019) Secured data collection with hardware-based ciphers for iot-based healthcare. IEEE Internet of Things Journal 6(1): 410-420.

4. Tian S, Yang W, Grange JML, Wang P, Huang W, et al. (2019) Smart healthcare: making medical care more intelligent. Global Health Journal 3: 62-65. 
5. Antonopoulos CP, Panagiotakopoulos TC, Panagiotou C, Tou- Liatos G, Koubias SA, et al. (2015) On developing a novel versatile framework for heterogeneous home monitoring WSN networks. EAI Endorsed Transactions on Pervasive Health and Tech- nology 1(1): e4.

6. Ali F, El-Sappagh SHA, Islam SMR, Ali A, Attique M, et al. (2021) $8^{\text {th }}$ acm international conference on pervasive technologies related to assistive environments. Future Generation Computer Systems 114: 23-43.

7. Awais M, Raza M, Ali K, Ali Z, Irfan M, et al. (2019) An internet of things based bed-egress alerting paradigm using wearable sensors in elderly care environment. Sensors 19(11): 2498.

8. Jiang L, Chen L, Giannetsos T, Luo B, Liang K, et al. (2019) Toward practical privacy-preserving processing over encrypted data in iot: An assistive healthcare use case. IEEE Internet of Things Journal 6(6): 10177-10190.

9. Panagiotou C, Panagiotakopoulos TC, Kameas A (2015) A multi: modal decision making system for an ambient assisted living environment. In 8th ACM International Conference on PErvasive Technologies Related to Assistive Environments (PETRA), p. 44.

10. Shahnaz A, Qamar U, Khalid A (2019) Using blockchain for electronic health records. IEEE Access 7: 147782-147795.

11. Sengupta J, Ruj S, Bit SD (2020) A comprehensive survey on attacks, security issues and blockchain solutions for iot and iiot. Journal of Network and Computer Applications 149: 102481.

12. Yue X, Wang H, Jin D, Li M, Jiang W (2016) Healthcare data gateways: Found healthcare intelligence on blockchain with novel privacy risk control. Journal of Medical Systems 40(10): 218.

13. Chen L, Lee W, Chang C, Choo KR, Zhang N (2019) Blockchain based searchable encryption for electronic health record sharing. Future Generation Computer Systems 95: 420-429.

14. McGhin T, Choo KR, Liu CZ, He D (2019) Blockchain in healthcare applications: Research challenges and opportunities. Journal of Network and Computer Applications 135: 62-75.

15. Vonitsanos G, Panagiotakopoulos T, Kanavos A, Maragoudakis M, Mylonas P (2021) Issues and challenges associated with blockchain in smart cities. In $16^{\text {th }}$ International Workshop on Semantic and Social Media Adaptation and Personalization (SMAP). IEEE.

16. Ferna'ndez-Carame's TM, Fraga-Lamas $P$ (2018) A review on the use of blockchain for the internet of things. IEEE Access 6: 32979-33001.

17. Khalilov MCK, Levi A (2018) A survey on anonymity and privacy in bitcoin-like digital cash systems. IEEE Communications Surveys and Tutorials 20(3): 2543-2585.

18. Khan MA, Salah K (2018) Iot security: Review, blockchain solutions, and open challenges. Future Generation Computer Systems 82: 395-411.

19. Khatoon (2020) A blockchain-based smart contract system for healthcare management. Electronics 9(1): 94.

20. Li X, Jiang P, Chen T, Luo X, Wen Q (2020) A survey on the security of blockchain systems. Future Generation Computer Systems 107: 841853.

21. Yli-Huumo J, Ko D, Choi S, Park S, Smolander K (2016) Where is current research on blockchain technology? - a systematic review. PloS One 11(10): e0163477.

22. Kumari A, Tanwar S, Tyagi S, Kumar N (2018) Fog computing for healthcare 4.0 environment: Opportunities and challenges. Computers \& Electrical Engineering 72: 1-13.

23. Perera S, Nanayakkara S, Rodrigo M, Senaratne S, Weinand R (2020) Blockchain technology: Is it hype or real in the construction industry? Journal of Industrial Information Integration 17: 100125
24. Vonitsanos G, Panagiotakopoulos T, Kanavos A, Tsakalidis AK (2021) Forecasting air flight delays and enabling smart airport services in apache spark. In $17^{\text {th }}$ International Conference on Artificial Intelligence Applications and Innovations (AIAI) 628: 407-417.

25. Ismail L, Materwala H, Zeadally S (2019) Lightweight blockchain for healthcare. IEEE Access 7: 149935-149951.

26. Shae Z, Tsai JJP (2017) On the design of a blockchain platform for clinical trial and precision medicine. In $37^{\text {th }}$ IEEE International Conference on Distributed Computing Systems (ICDCS), pp. 1972-1980.

27. Zhao G, Liu S, Lopez C, Lu H, Elgueta S, et al. (2019) Blockchain technology in agri-food value chain man- agement: A synthesis of applications, challenges and future research directions. Computers in Industry 109: 83-99.

28. Agbo CC, Mahmoud QH, Eklund JM (2019) Blockchain technology in healthcare: A systematic review. In Healthcare 7(2): 56.

29. Alonso SG, Arambarri J, Coronado ML, Torre D'ıez I de la (2019) Proposing new blockchain challenges in ehealth. Journal of Medical Systems 43(3): 64 .

30. Boulos MNK, Wilson JT, Clauson KA (2018) Geospatial blockchain: Promises, challenges, and scenarios in health and healthcare. International Journal of Health Geographics 17: 25.

31. Ho“lbl M, Kompara M, Kamisalic A, Zlatolas LN (2018) A systematic review of the use of blockchain in healthcare. Symmetry 10(10): 470.

32. Siyal AA, Junejo AZ, Zawish M, Ahmed K, Khalil A, et al. (2019) Applications of blockchain technology in medicine and healthcare: Challenges and future perspectives. Cryptography 3(1): 3.

33. Krania A, Statiri M, Kanavos A, Tsakalidis AK (2017) Internet of things services for healthcare systems. In $8^{\text {th }}$ International Conference on Information, Intelligence, Systems \& Applications (IISA), p. 1-6.

34. Mettler M (2016) Blockchain technology in healthcare: The revolution starts here. In $18^{\text {th }}$ IEEE International Conference on e-Health Networking, Applications and Services (Healthcom), p. 1-3.

35. Garay JA, Kiayias A, Leonardos N (2015) The bitcoin backbone protocol: Analysis and applications. In $34^{\text {th }}$ Annual International Conference on the Theory and Applications of Cryptographic Techniques (EUROCRYPT) 9057: 281-310.

36. Gervais A, Karame GO, Capkun V, Capkun S (2014) Is bitcoin a decentralized currency? IEEE Security and Privacy 12(3): 54-60.

37. Nakamoto S (2008) Bitcoin: A peer-to-peer electronic cash system. Decen- tralized Business Review, pp. 21260.

38. Cai Y, Zhu D (2016) Fraud detections for online businesses: A perspective from blockchain technology. Financial Innovation 2(1): 1-10.

39. Kiyomoto S, Rahman MS, Basu A (2017) On blockchain-based anonymized dataset distribution platform. In $15^{\text {th }}$ IEEE International Conference on Software Engineering Research, Management and Applications (SERA), p. 85-92.

40. Roehrs A, Da Costa CA, Da Rosa Righi R (2017) Omniphr: A distributed architecture model to integrate personal health records. Journal of Biomedical Informatics 71: 70-81.

41. Azaria A, Ekblaw A, Vieira T, Lippman A (2016) Medrec: Using blockchain for medical data access and permission management. In $2^{\text {nd }}$ International Conference on Open and Big Data (OBD), p. 25-50.

42. Samaniego M, Deters R (2016) Hosting virtual iot resources on edgehosts with blockchain. In IEEE International Conference on Computer and Information Technology (CIT), pp. 116-119. 
43. Yaqoob I, Salah K, Jayaraman R, Al-Hammadi Y (2021) Blockchain for healthcare data management: Opportunities, challenges, and future recommendations. Neural Computing and Applications, p. 1-16.

44. Wang S, Wang J, Wang X, Qiu T, Yuan Y, et al. (2018) Blockchainpowered parallel healthcare systems based on the ACP approach. IEEE Transactions on Computational Social Systems 5(4): 942-950.

45. Khan WA, Khattak AM, Hussain M, Amin MB, Afzal M, et al. (2014) An adaptive semantic based mediation system for data interoperability among health information systems. Journal of Medical Systems 38(8): 28.

46. Reisman M (2017) Ehrs: The challenge of making electronic data usable and interoperable. Pharmacy and Therapeutics 42(9): 572-575.

47. Dagher GG, Mohler J, Milojkovic M, Marella PB (2018) Ancile: Privacypreserving framework for access control and interoperability of electronic health records using blockchain technology. Sustainable Cities and Society 39: 283-297.

48. Khan FA, Asif M, Ahmad A, Alharbi M, Aljuaid H (2020) Blockchain technology, improvement suggestions, security challenges on smart grid and its application in healthcare for sustainable development. Sustainable Cities and Society 55: 102018

\section{ISSN: 2574-1241}

DOI: 10.26717/BJSTR.2021.40.006423

Andreas Kanavos. Biomed J Sci \& Tech Res

(C) This work is licensed under Creative BY Commons Attribution 4.0 License

Submission Link: https://biomedres.us/submit-manuscript.php
49. Vazirani AA, O’Donoghue O, Brindley D, Meinert E (2020) Blockchain vehicles for efficient medical record management. NPJ Digital Medicine 3(1): 1-5.

50. Kassab MH, DeFranco J, Malas T, Laplante P, Destefanis G, et al. (2019) Exploring research in blockchain for healthcare and a roadmap for the future. IEEE Transactions on Emerging Topics in Computing.

51. Kumar T, Ramani V, Ahmad I, Braeken A, Harjula E, et al. (2018) Blockchain utilization in healthcare: Key requirements and challenges. In $20^{\text {th }}$ IEEE International Conference on e-Health Networking, Applications and Services (Healthcom), p. 1-7.

52. Xia Q, Sifah EB, Asamoah KO, Gao J, Du X, et al. (2017) Medshare: Trustless medical data sharing among cloud service providers via blockchain. IEEE Access 5: 14757-14767.

53. Linn LA, Koo MB (2016) Blockchain for health data and its potential use in health it and health care related research. In ONC/NIST Use of Blockchain for Healthcare and Research Workshop, p. 1-10.

54. Stagnaro C (2017) White paper: Innovative blockchain uses in health care. Freed Associates.

55. Beck R (2018) Beyond bitcoin: The rise of blockchain world. Computer 51(2): 54-58.

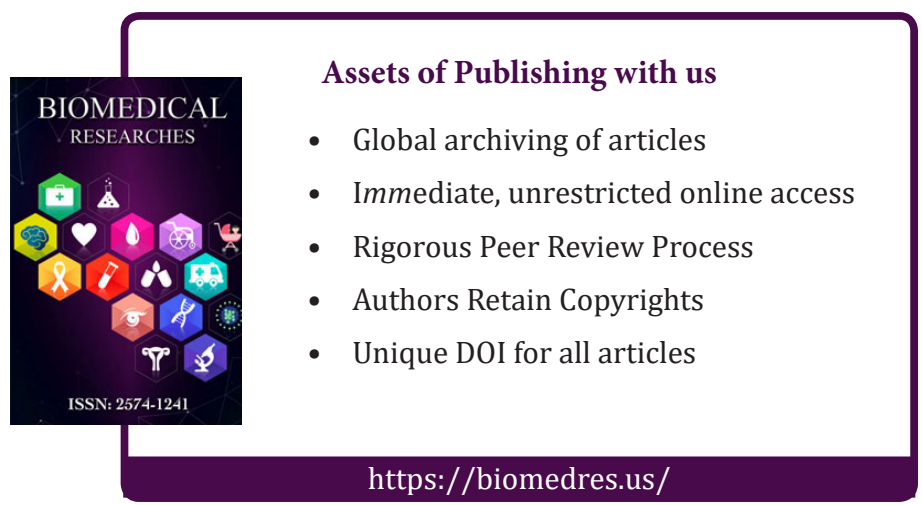

